\title{
Creating common operational pictures for disaster response with collaborative work
}

\author{
T. Chen, G. Su \& H. Yuan \\ Institute of Public Safety Research, \\ Department of Physics Engineering, Tsinghua University, China
}

\begin{abstract}
People need to understand the kinds of disaster information available from the multiple participants involved and respond to complex situations in disaster management. This paper presents a method of constructing common operational pictures to achieve situation awareness during disaster response among the emergency response agencies. The method of common operational pictures is featured with the visualization and collaborative work of current undergoing response measures and future state prediction provided by computational disaster models. It was visualized by integrating base map, disaster influence, future state prediction and resource allocation supported by GIS technologies. The technical architecture was realized with GIS services and data fusion technologies in a network centric system.
\end{abstract}

Keywords: GIS, common operational pictures, disaster response, collaborative work.

\section{Introduction}

People have been fighting with all kinds of disasters for centuries. With the fast development of human society, lifeline systems, transportation systems, various industrial facilities and concentration of population in cities have tremendously increased not only vulnerability but also risk of our world.

Disaster management, which covers multiple research areas and involves various aspects, is a very complex and hot topic to both scientists and government officers. Recent years, our world suffered several catastrophes such as China's May 12th Wenchuan earthquake in 2008 and Japan's March 11th earthquake/tsunami in 2011. These disasters have demonstrated the 
complexity of disaster management. Managing disasters need collaborative work of multiple stakeholders and achieve situation awareness. Emergency operations are mostly carried out by government agencies. When the incident is of significance and complexity, information should be shared between multiple agencies and collaborative work is needed.

The Common Operating Pictures (COP) is a military acronym synonymous first developed by US Army. The aim of COP is to seeing and understanding. COP [1] is a single identical display of relevant information shared by more than one command. A common operational picture facilitates collaborative planning and assists all echelons to achieve situational awareness. In disaster management, situation awareness (SA) is critical to all involved agencies and decision makers. In disaster response C2 (Command \& Control) system [2], the COP concept is also of great importance. Modern information technology such as Geographic Information System (GIS) and Web-based technologies has been used to support disaster management.

Geospatial information is crucial to decision-making and situation awareness. It is widely acknowledged that GIS plays a significant role in providing informational and analytical tools to disaster management [3-5]. It has been widely used for decision-making, which provides the capability of visualization of hazard map and spatial expression of resource location as well as command intentions. In disaster management, GIS based COP must be visualized and displayed to involved parties that have different roles and responsibilities, such as planning, field operation, resource allocation and logistics. These parties should be connected together by a network centric disaster response system.

\section{COP needs collaborative work}

Disaster response needs collaborative work. Under the situation of significant disasters, multiple governmental departments are usually involved and take different responsibilities in response. Vertically, several response levels including local communities, city government, state government and mobile command post in the field, will be involved according to the significance of the incident. Horizontally, several government departments such as police, medical service, fire and transportation will also be involved according to the discipline of the disaster. They need to work with each other closely and efficiently. The question is how to maintain unified situation awareness and command both vertically and horizontally for all of them (shown in Figure 1). This poses an important collaboration issue in disaster management.

Disaster management related agencies usually work in distributed places. Rescue teams and resources are also dispatched to the field of emergency operations. Disaster and response information should be fused from and share to the most extensive stakeholders, as well as to the foremost parties. The capability for different users to access GIS services, base maps, base layers and data from remote sites is crucial. Only a network centric system can bring them to the virtual and cyber consultation space wherever they are physically located. 
Disaster response COP offers a comprehensive, map based solution for disaster response that enables collaboration across organizational boundaries. COP may facilitate the sharing of the right information with the right people. The heightened situational awareness provided by the COP will keep everybody on the same page and synchronized for maximum effect.

The interaction between multiple users comprises textual comments, related multimedia documents (images, text, videos etc.) and geographic objects (points, lines, polygons), which can be directly posted into the map. The whole map view can be made available to other users via a web based work platform, whereby access can optionally be restricted to user groups or single users.

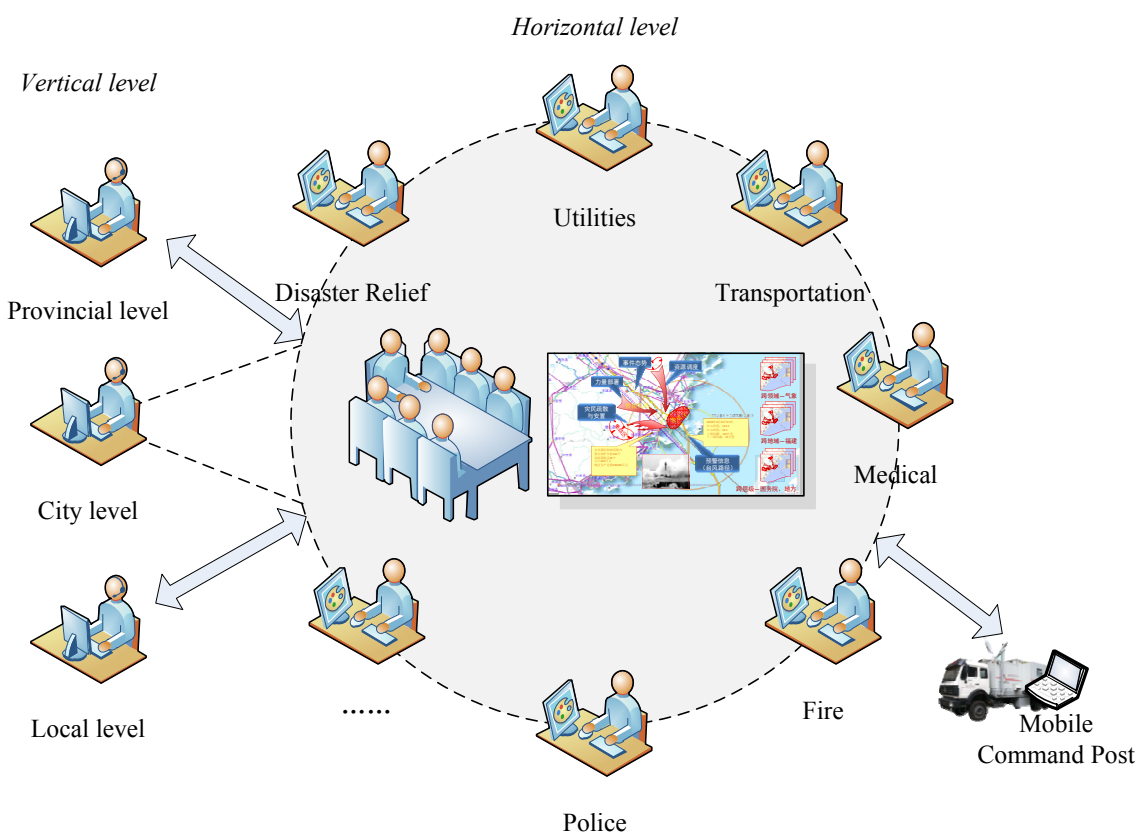

Figure 1: Collaborative work to create disaster COP.

\section{Disaster information fusion, sharing and prediction}

Information is the key to disaster response decision making for unified command and section command. Disaster response needs to collect and share various kinds of information, both static background data and real-time disaster situation. Those related information, including the accurate disaster location, the significance, the impact area, the casualties and the field visual information should be transferred vertically from the field command post to the off-site command center. The command center usually has databases support, risk analysis capabilities, disaster model simulation capabilities and should hold the whole picture of the situation. On the other hand, involved departments hold 
peculiar data and should share their information horizontally to each other. Each participant department need to provide the information of resource dispatching, impact prediction, trend analysis, action plans, on-scene video, etc.

Disaster response COP will provide a much needed mapping component and displays real time operational information and combines it with the traditional static GIS layers, as shown in Figure 2. First responders, decision makers and related stakeholders have a much richer set of situational awareness data available to them so that they can plan and execute responses more timely and effectively.

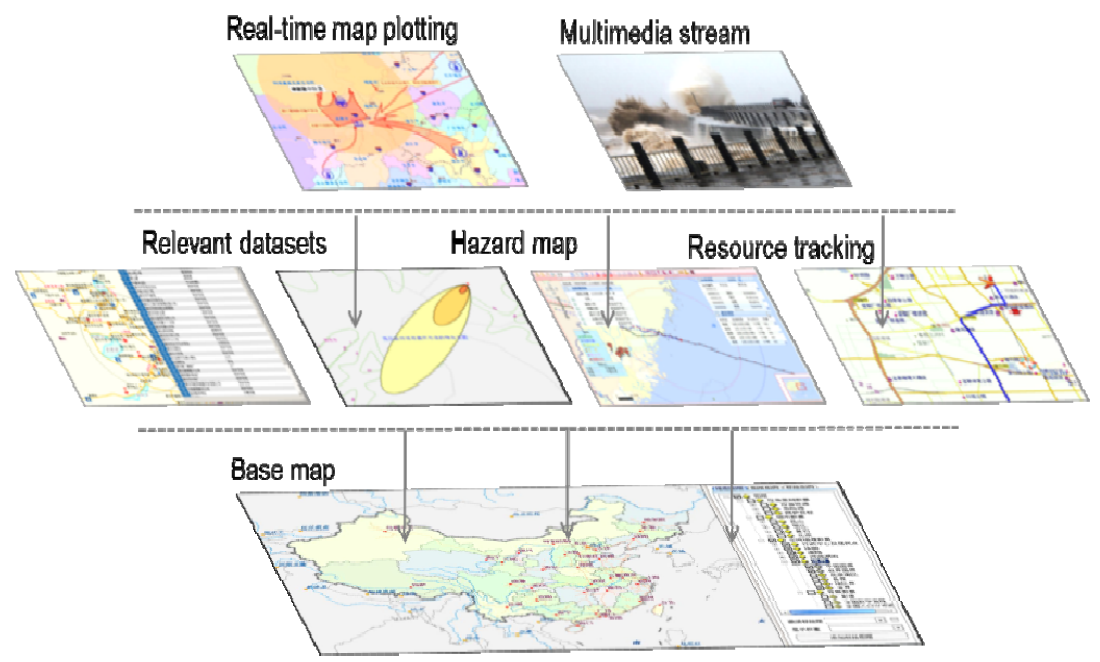

Figure 2: $\quad$ Schematic view of COP.

\subsection{Data fusion and sharing}

In disaster response COP, the following kinds of information should be included:

a. Base map. Through the geo-data management capabilities of GIS, the base map will include the digital line graph, digital terrain, RS images as well as residential areas, roads, rivers, lakes, place names;

b. Distribution of public safety related datasets. When disaster strikes, the points of concern will be searched from the databases and displayed on the base map. Those include population, public safety resources, civilian resources, critical infrastructure, as well as other national assets of importance. When these types of assets are inventoried and accessible through GIS, the appropriate resources (proximity to an emergency) can be brought in for timely response;

c. Incident future state prediction by hazard map and hot zones. The affected areas caused by an incident such as a spatially distributed wind field driving the dispersion of a toxic gas. This also includes spatially distributed impacts 
and areas of varying vulnerability identified by the risk analysis models. GIS based visualization module provides the graphic representation of the geographic maps, model output data and scenario. Their distribution may be visualized using coordinates of points, lines or areas and their evolution over time may be visualized using versicolor overlay layers of nephogram on GIS;

d. Updating real-time situation reports on disaster loss such as casualties and property loss, and resource allocation such as rescue team and relief goods. These are the basic information used to take corresponding operations and logistics and should be keep up to date and marked up on map. The spatial distribution of emergency response teams such as police, medical teams, fire and urban search and rescue teams should be include in the disaster response COP;

e. Decision and rescue plans. The intension of an incident action plan can be drawn on the disaster response COP map, indicating the people evacuation route, rescue teams' staging areas and dispatching activities, etc.

f. Other real-time multimedia feedback. The COP should include surveillance video and photos from on-site command post, airborne cameras and fixed street cameras.

The above information will be classified and displayed on different map layers made up before an incident or dynamically generated during the progress of response operations.

\subsection{COP architecture}

The architecture of disaster response COP provides a platform for the disaster response system to interact and collaborate with each other. It is provided with a group of web services that enable situation awareness through web browsers or other client based graphic user interfaces.

The architecture of disaster response COP is shown in Figure 3.

Disaster response COP come from technical solutions on GIS and non-GIS data fusion, which includes the existing data sources and real-time information came into the system:

(1) By creating a geodatabase model, integrates data exist in multiple disparate databases to build a common base map, then shares the map via network;

(2) By providing a Service Oriented Architecture (SOA) implementation, ingest other information through Web Services;

(3) By providing collaborative map plotting, instant text message and web video conversation capability, brings different users at distributed places into a cyber space and instant sharing information though network;

(4) By creating data exchanging interface, fuses data from disparate data sources of different departments, then permits re-use of existing data assets;

(5) By developing interfaces to resource tracking system, provide on-demand linking of location data to resources, such as GPS;

(6) Support OGC and ISO standards, such as Web Map Service (WMS), and recent Web feed standard. 


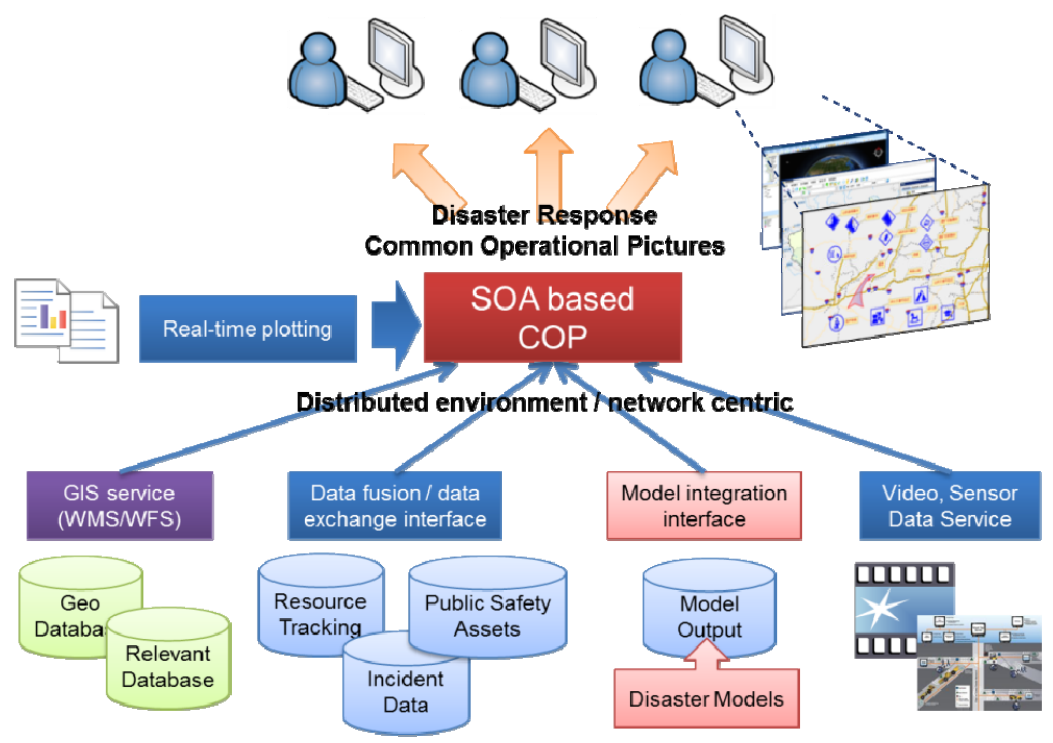

Figure 3: Architecture of COP.

\subsection{Integrating computational models}

Besides the information about what happened, disaster response needs future state prediction of what would happen. It is very important when response to a disaster. Here, COP not only hub the information but also provide future state prediction based on the computational disaster models that are widely used in disaster evolvement and risk analysis. Usually the command center, one of the departments or the expertise group has this kind of risk analysis capability to generate a future state hazard map.

Unlike the battlefield, disaster can be modeled and predicted in many ways with computational models. In disaster response, disasters are analyzed and coped with in various aspects, such as disaster evolution analysis, impact on infrastructures, human behavior and rescue actions. Those aspects are now supported with many computational models [6, 7]. Programs for automated forecasts, modeling, warning, risk evaluation and evacuation modeling can be used for disaster response. These models are developed to explain and image the disaster mechanism and its impact.

The GIS focuses on visualizing different types of information and offering spatial analysis capabilities. Moreover, GIS will provide more advanced analysis functionality such as disaster modeling and simulating capability. Computer models that simulate disasters require geographic database and topic-oriented databases. GIS based visualization module provides the graphic representation of the geographic maps, model output data and scenario. 


\section{Conclusion}

People need to understand kinds of incident information from multiple participants involved in and respond to complex situation in disaster management. GIS based disaster response COP will help to respond quickly to events with geospatial capabilities for more efficient operations between involved participants. Disaster response COP will help to enhance situation awareness in response to significant disasters. It is also featured with the capability of integrating future state prediction provided by computational models.

One scenario is to response to a typhoon incident in China, several departments such as meteorological, water resource, land resource, civil affair, police, transportation and the city command center will contribute to the disaster response COP. For example, meteorological bureau will provide the affecting area using weather data, the route of typhoon, wind speed circle and the rain forecast onto the COP map. Water resource department will forecast based on the above information to predict flood or waterlogging. Land resource department will find out the landslide hazard and mark up on the COP map. The city command center will find out the influence in the vicinity, population, critical objects etc. When all the information are fused and shared using disaster response COP through collaborative work, it will allow for a real-time situational awareness making emergency and rescue actions much more efficient.

Summarizing, the scope of the disaster response COP concept can be described as a ubiquitous operation and decision support framework allowing collaborative work, producing a real-time availability of data such as maps, datasets, geo-objects, multimedia or comments.

\section{Acknowledgement}

Project 71073093 supported by NSFC.

\section{References}

[1] CJCSI 3151.01A. (2003) Global Command and Control System Common Operational Picture Reporting Requirements.

[2] Junget, E., Hallberg, N. (2009) Capabilities of C2 Systems for Crisis Management in Local Communities. Proceedings of the 6th International ISCRAM Conference. Gothenburg, Sweden.

[3] Chang, N.B., Wei, Y.L., Tseng, C.C. and Kao, C.YJ. (1997)The design of a GIS-based decision support system for chemical emergency preparedness and response in an urban environment. Computers, Environment and Urban Systems. 21, 1, 67-94.

[4] Johnson, R. (2000) GIS Technology for Disasters and Emergency Management. An ESRI White Paper.

[5] Wang, J.P., Ma, J.P. (2004) Research of Urban Emergency Rescue System Based on GIS. Geospatial Information. 2, 3, 25-27. 
[6] Zografos, K.G., Vasilakis, G.M., Giannouli, I.M. (2000) Methodological framework for developing decision support systems(DSS) for hazardous materials emergency response operations. Journal of Hazardous Materials. 71, 503-521.

[7] Chen, T., Yuan, H.Y., Yang, R., Chen, J.G. (2008) Integration of GIS and Computational Models for Emergency Management. Proceedings 2008 International Conference on Intelligent Computation Technology and Automation. Changsha, China. 